\title{
Functional respiratory imaging: heterogeneity of acute exacerbations of COPD
}

This article was published in the following Dove Press journal:

International Journal of COPD

\section{Wouter H van Geffen ${ }^{1,2}$ \\ Bita Hajian ${ }^{3}$ \\ Wim Vos ${ }^{4}$ \\ Jan De Backer ${ }^{4}$ \\ Anthony Cahn ${ }^{5}$ \\ Omar S Usmani ${ }^{6}$ \\ Cedric Van Holsbeke ${ }^{4}$ \\ Massimo Pistolesi ${ }^{7}$ \\ Huib AM Kerstjens ${ }^{2}$ \\ Wilfried De Backer ${ }^{3}$}

'Department of Respiratory Medicine, Medical Centre Leeuwarden, Leeuwarden, the Netherlands; ${ }^{2}$ Department of Pulmonary Diseases, University Medical Center Groningen, Groningen Research Institute for Asthma and COPD, University of Groningen, the Netherlands; ${ }^{3}$ Department of Pulmonary Diseases, Antwerp University Hospital, Antwerp, Belgium; ${ }^{4}$ FLUIDDA nv, Kontich, Belgium; ${ }^{5} \mathrm{GlaxoSmithKline} \mathrm{R \& D,}$ Stevenage, UK; ${ }^{6}$ Faculty of Medicine, National Heart \& Lung Institute, Imperial College London, London, UK; ${ }^{7}$ Department of Experimental and Clinical Medicine, Section of Respiratory Medicine, University of Florence, Florence, Italy
Correspondence: Wouter $\mathrm{H}$ van Geffen Department of Respiratory Medicine, Medical Centre Leeuwarden, Henri Dunantweg 2, 8934 AD Leeuwarden, the Netherlands

Tel +3I 582866666

Email wouter.van.geffen@znb.nl
Background: Exacerbations of COPD are a major burden to patients, and yet little is understood about heterogeneity. It contributes to the current persistent one-size-fits-all treatment. To replace this treatment by more personalized, precision medicine, new insights are required. We assessed the heterogeneity of exacerbations by functional respiratory imaging (FRI) in 3-dimensional models of airways and lungs.

Methods: The trial was designed as a multicenter trial of patients with an acute exacerbation of COPD who were assessed by FRI, pulmonary function tests, and patient-reported outcomes, both in the acute stage and during resolution.

Results: Forty seven patients were assessed. FRI analyses showed significant improvements in hyperinflation (a decrease in total volume at functional residual capacity of $-0.25 \pm 0.61 \mathrm{~L}$, $p \leq 0.01)$, airway volume at total lung capacity $(+1.70 \pm 4.65 \mathrm{~L}, p=0.02)$, and airway resistance. As expected, these improvements correlated partially with changes in the quality of life and in conventional lung function test parameters. Patients with the same changes in pulmonary function differ in regional disease activity measured by FRI.

Conclusion: FRI is a useful tool to get a better insight into exacerbations of COPD, and significant improvements in its indices can be demonstrated from the acute phase to resolution even in relatively small groups. It clearly visualizes the marked variability within and between individuals in ventilation and resistance during exacerbations and is a tool for the assessment of the heterogeneity of COPD exacerbations.

Keywords: COPD exacerbations, FRI, hyperinflation, chronic obstructive pulmonary disease, symptoms, heterogeneity

\section{Introduction}

COPD is a disease with an enormous personal and societal burden. Considerable proportions of the morbidity, mortality, and costs of this disease are related to exacerbations of COPD. ${ }^{1}$ Relatively little is known about exacerbations, and the quest to effectively prevent and manage them continues. Medical treatment of acute exacerbations routinely consists of steroids, bronchodilators, antibiotics, additional oxygen, and sometimes assisted ventilation. Currently, a one-size-fits-all treatment for acute exacerbations is provided in most clinics. We and many others believe that this should urgently be replaced by more personalized, precision medicine.

Some tools have been developed to more adequately monitor the start and resolution of an exacerbation. Progress is definitely being made in the field of patient-reported

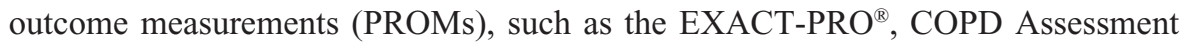
Test (CAT), and Clinical COPD Questionnaire (CCQ). ${ }^{2,3}$ Traditionally, COPD patients are monitored by pulmonary function tests such as the forced expiratory volume in 1 second $\left(\mathrm{FEV}_{1}\right)$. However, especially during exacerbations, changes in $\mathrm{FEV}_{1}$ are small 
and correlate poorly with patient-reported complaints such as dyspnea and with the response to medication. ${ }^{4-6}$ Both clinicians and researchers struggle with this issue on a daily basis. Therefore, new and more informative correlates of disease severity and response are eagerly awaited to allow individualized therapy, especially during exacerbations. A model for a differential approach depending on the inflammatory status has been developed, where eosinophilic inflammation and bacterial infections help guide therapy. However, this does not address viral infections. ${ }^{7,8}$ Furthermore, we believe that hyperinflation, which has been shown to be important in guiding therapy in a stable state, should also be addressed during acute exacerbations. ${ }^{1,9-12}$

Hyperinflation is caused by trapping of air during expiration, due to peripheral airway obstruction. It increases functional residual capacity (FRC) in such a way that inspiratory capacity (IC) decreases, resulting in increased dyspnea and limitation of exercise capacity..$^{5,10,13-15}$ Some data about hyperinflation during exacerbations are available, showing that it increases. ${ }^{5,15}$ Whether the increase in hyperinflation during exacerbations is specifically caused by changes in the peripheral airways and decreased expiratory time at times of greater demand remains unknown. Hyperinflation during exacerbations has been studied mostly by IC, yielding limited data, on aggregated level only. The result of this measurement is driven by several more regional factors, contributing differently between patients. Insightful knowledge of changes in hyperinflation during exacerbations is currently lacking, which is most mechanistically unsatisfactory, and hampers the development of personalized treatment.

Next to hyperinflation, increased airway resistance has been observed in exacerbations. ${ }^{5,16,17}$ Airway resistance correlates with dyspnea and recovery and stable state bronchodilator response. ${ }^{5,16,17}$ Until this point, the heterogeneity of airway resistance has not been measured in exacerbations, although a technique to measure this value has been developed in the stable state of COPD. ${ }^{18}$ Clinical consequences of regional differences in airway resistance thus far are not available, perhaps since this technique has been developed only recently.

This promising new computational technique to better understand the mechanisms of acute exacerbations is called functional respiratory imaging (FRI). ${ }^{18}$ Based on high-resolution computed tomography (HRCT) scans, 3-dimensional models of airways and lung are extracted and used for computational fluid dynamics (CFD) simulations. The technique has been extensively tested in stable COPD and other diseases and is clinically validated. ${ }^{18-21}$ One of the core features of FRI is that local measurements of lobar volumes, airway volumes, and airway resistance can be performed, while for instance the $\mathrm{FEV}_{1}$ incorporates the whole respiratory system into a single number. The usefulness of FRI has been proven in assessing hyperinflation, airway resistance, and airway diameter during stable state of COPD, but no data are available in the acute setting. ${ }^{20,22,23}$

This study was designed to test whether FRI has additional value on top of assessing conventional lung function tests and patient-reported outcomes (PROs) in the monitoring of patients with an exacerbation of COPD. We hypothesized that it is feasible to measure FRI also in the setting of acute exacerbations of COPD and that changes in FRI parameters correlate with changes in lung function parameters and PROs during resolution of COPD exacerbations, especially for $\mathrm{FEV}_{1}$ and hyperinflation. The inherent regional aspects of FRI should allow the detection of heterogeneity in exacerbating COPD patients.

\section{Methods}

The trial was designed as an international multicenter prospective cohort study. Patients were recruited from 3 hospitals in Belgium, Italy, and the Netherlands. Protocols and patient information were all approved by the designated medical ethics committees (Comite voor medische Ethiek, Universiteit Ziekenhuis Antwerpen, Comitato Etico [per la sperimentazione clinica dei medicinali] dell'Azienda Ospedaliero Universitaria Careggi, and Medische Ethische Toetsingscommissie, Universitair Medisch Centrum Groningen). The trial was registered as NCT01684384 at www. ClinicalTrials.gov.

Patients were recruited at the start of an exacerbation, when written informed consent was obtained. The treatment of the exacerbation was applied as usual care, according to the Global initiative for chronic Obstructive Lung Disease (GOLD) guidelines, and consisted at least of additional bronchodilation and systemic corticosteroids. ${ }^{24}$

A patient was eligible for inclusion only if all of the following criteria applied: aged $\geq 40$ years; diagnosed with COPD with postbronchodilator $\mathrm{FEV}_{1}$ /forced expiratory vital capacity (FVC) $<70 \%$ and postbronchodilator $\mathrm{FEV}_{1}<80 \%$ predicted as documented in the last 5 years; and having a COPD exacerbation defined as an acute change in the patient's baseline dyspnea, cough, and/or sputum that is beyond normal day-to-day variations and that necessitated the administration or doubling of systemic corticosteroid treatment.

Patients were excluded from participation if any of the following criteria applied: pregnant or lactating women; 
patients diagnosed with asthma; and patients with pneumonia as defined radiologically at the start of the exacerbation, a history of or the presence of lung cancer, and an indication for noninvasive ventilation. Patients who were unlikely to comply with the protocol or unable to understand the nature, scope, and possible consequences of the study were also excluded, as were patients who had received any investigational new drug within the last 4 weeks prior to visit 1 . Due to the recommendations of the medical ethics committee in the Netherlands, patients with a weight $>110 \mathrm{~kg}$ were excluded, in the Netherlands only.

\section{Measurements PROs}

All measurements detailed below were obtained within 5 days of the start of the exacerbation, and repeated at a stable state, ie, day 42 , provided that the exacerbation had resolved as judged by their physician. Otherwise, the stable state measurements were postponed.

Health-related quality of life and dyspnea score were measured by validated PROMs: the Saint George's Respiratory Questionnaire (SGRQ), CCQ, CAT, and Modified Medical Research Council (mMRC) dyspnea scale. ${ }^{3,25}$

\section{Lung function}

$\mathrm{FEV}_{1}$, IC, FVC, residual volume, total lung capacity (TLC) by plethysmography, FRC, and airway resistance (by plethysmography [Raw]) were measured at both visits, according to the recommendations of the European Respiratory Society (ERS) guidelines with the accompanying reference values. ${ }^{26}$ All lung function tests were repeated, until 3 technically acceptable measurements had been made, with a maximum of 8 measurements. There was no withholding of bronchodilator treatment for the lung function measurements.

\section{CT}

During the exacerbation, and in a clinically stable state, HRCT scans at FRC and TLC during breath-hold were performed. In order to ensure that the scans were taken at the correct lung function level, FRC and TLC were ensured by lung function technician coaching with the aid of a spirometer. Specific CT settings are reported in the Supplementary material.

Postprocessing of the CT data included segmentation of the airway tree (Mimics; Materialise, Leuven, Belgium). Three-dimensional models of airways and lungs were extracted from scan and were used for CFD simulations. FRI, a clinically validated computational workflow, was used to perform measurements of lobar volumes (by FRI [iVlobe] " $i$ " designates "imaging"), a parameter for lung hyperinflation. It was also used to assess airway volumes (by FRI [iVaw]) and airway resistances (by FRI [iRaw]; Figure 1). It has the potential to measure all these parameters at all lobes, at different airway generations and time points. We predefined the most important parameters: changes from exacerbation to stable state in the total score of both lungs for the parameters iVlobe, iVaw, and iRaw. Based on the clinical relevance for

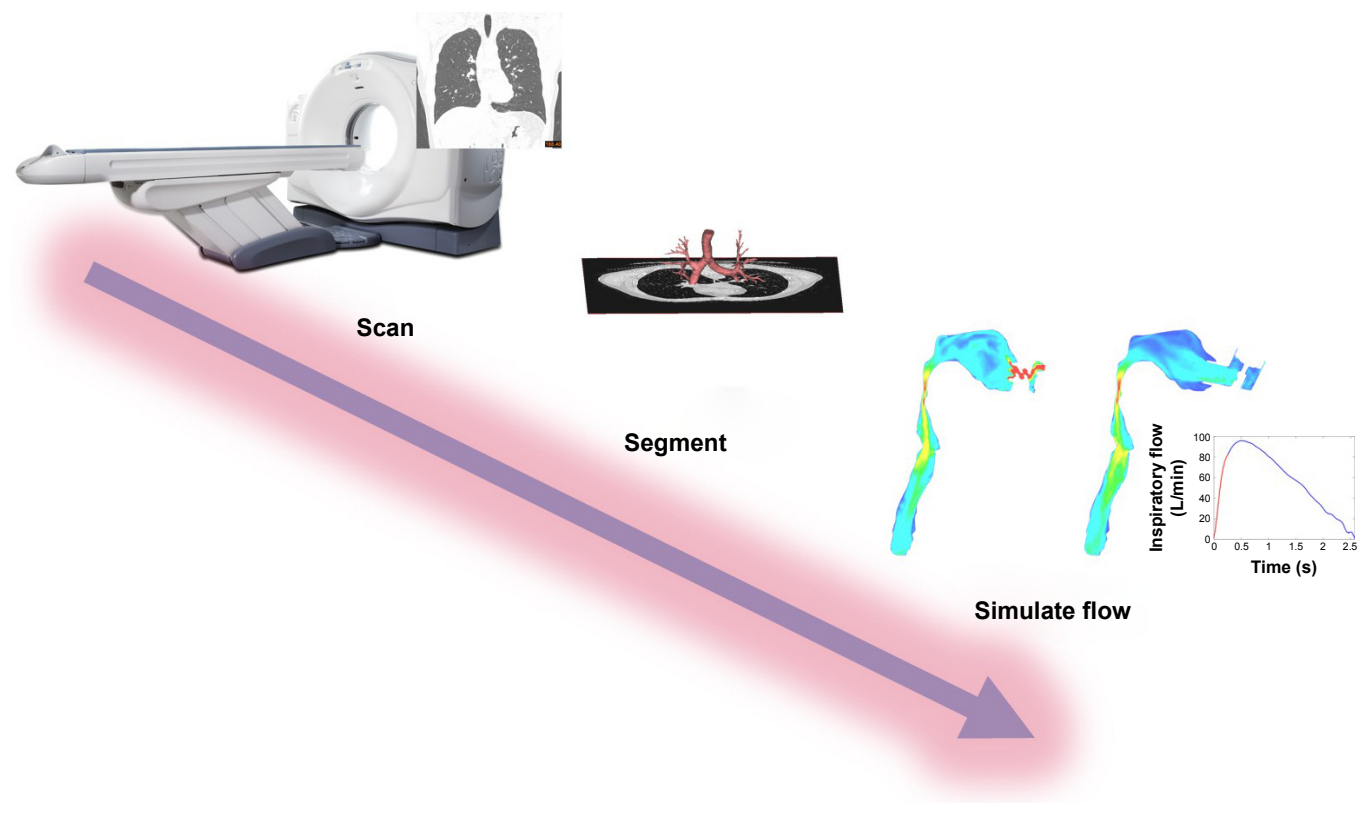

Figure I Overview of the FRI concept. Patients are first scanned. Then, the CT images are segmented, and the rendering of the airways is calculated. Then, a 3-dimensional model of the lung is developed. Finally, flow is simulated in this model using CFD techniques. ${ }^{18}$

Abbreviations: CFD, computational fluid dynamics; CT, computed tomography; FRI, functional respiratory imaging. 
each FRI parameter, measurements at FRC or TLC level were selected.

\section{Analyses}

\section{Study outcomes}

The primary goal was to assess, during the resolution of an exacerbation, the strength of the association between change in lung function and change in parameters measured by FRI. The secondary goal was to assess the strength of the association between the changes in the same FRI parameters and SGRQ, CAT, mMRC, and CCQ.

\section{Statistical analyses}

A power calculation was based on an estimated correlation coefficient of 0.7 for the primary objectives with $95 \%$ confidence interval of $0.52-0.80$. The number of subjects needed to achieve $80 \%$ power with $\alpha$ of 0.05 was 50 (2-sided sample size calculation for a Pearson correlation analysis derived by Power and Precision ${ }^{\mathrm{TM}} 4.0$ [Biostat, Englewood, NJ, USA]).

The analyses were performed by using $\mathrm{R}$ Version 3.2.3 (The R Foundation for Statistical Computing, Vienna, Austria). All continuous variables (imaging-based volumes and CFD-based resistances, lung function parameters, and PROMs) were tested for normality using Shapiro-Wilk $W$ tests and transformed if necessary and successful.

Paired Student's $t$-tests were used to evaluate the changes in these parameters from exacerbation to recovery. These tests were also used to check for the segmentation reproducibility of the FRI measurements. Two-sided testing was performed.

Correlations between the continuous variables (the changes) were examined using multiple regression analysis techniques. The analysis of variance was used to fit the model, and goodness-of-fit statics were performed. A $p$-value $<0.05$ was defined as statistically significant. Parameters are reported as mean \pm standard deviation for descriptive statistics and \pm standard error of the mean for evaluative statistics.

\section{Results}

Between October 2012 and August 2014, a total of 54 patients were enrolled in this trial: 38 patients in Belgium, 6 patients in the Netherlands, and 10 patients in Italy. Table 1 presents the main characteristics of the recruited patients. Forty-seven patients were evaluable due to 7 dropouts ( 3 participants died, 3 withdrew consent, and 1 was lost to follow-up). All the participants were Caucasian.
Table I Patient characteristics $(n=47)$

\begin{tabular}{ll}
\hline Patient characteristics & Value \\
\hline Sex (male/female) & $27 / 20$ \\
Age (years) & $68.0 \pm 9.9$ \\
GOLD stage 2/3/4 & $19 / 22 / 6$ \\
Current smokers/ex-smokers/never smokers & $13 / 33 / 1$ \\
Pack-years & $46.5 \pm 22.2$ \\
Treatment in outpatient setting/hospital setting & $9 / 38$ \\
Days between exacerbation and stable state measurements & $55.1 \pm 22.7$ \\
\hline
\end{tabular}

Notes: Results are shown as mean \pm standard deviation or $n$. Demographics presented were measured during screening, and the lung function data presented in this table were measured at stable state.

Abbreviation: GOLD, Global initiative for chronic Obstructive Lung Disease.

\section{Changes from exacerbation to stable state}

Parameters of FRI improved from the acute phase to resolution of the exacerbation (Table 2; Figures 2 and 3). A significant improvement was found in hyperinflation, ie, a decrease in total volumes (iVlobe, total) at FRC of $-0.25 \pm 0.61 \mathrm{~L}(p \leq 0.01)$. Similarly, iVaw (total) at TLC increased, $1.70 \pm 4.65 \mathrm{~L}(p=0.02)$, though not at FRC. The iRaw at both FRC and TLC levels decreased significantly during recovery (Table 2).

Significant improvements were also found in $\mathrm{FEV}_{1}, \mathrm{IC}$, and FRC (Table 2), as well as in airway resistance measured by plethysmography. Patients reported significant improvements in health-related quality of life (SGRQ, CCQ, and CAT). All improvements were greater than the minimal clinical important difference and therefore also clinically significant.

\section{Correlations of FRI parameters with lung function and PROMs}

The strength of the association between the changes from exacerbation to stable state in conventional lung function parameters $\left(\mathrm{FEV}_{1}, \mathrm{IC}\right.$, and Raw) and changes in parameters measured by FRI (iVaw, iVlobe, and iRaw) was assessed. Change in $\mathrm{FEV}_{1}$ was correlated with specific airway volumes (by FRI [siVaw], FRC; $p=0.02, r=0.34$ ). The change in airway resistance measured by plethysmography was correlated with change in airway resistance measured by FRI (iRaw, $p=0.04$ ). The changes in $\mathrm{FEV}_{1}$, specific airway resistance measured by pulmonary function test, and IC were not correlated with the changes in iVaw, specific airway resistance measured by FRI (siRaw), and iVlobe. Changes in CCQ and CAT were correlated with changes in FRI-based siRaw. Changes in mMRC were correlated with changes in siVaw. By contrast, the changes in PROs did not correlate significantly with changes in iVlobe or iVaw (Table 3). 
Table 2 Change from exacerbation to stable state in lung function and FRI parameters

\begin{tabular}{|c|c|c|c|c|}
\hline Parameters & At exacerbation & At stable state & Mean change & Significance \\
\hline $\mathrm{FEV}_{1}(\mathrm{~L})$ & $1.15 \pm 0.43$ & $1.31 \pm 0.50$ & $0.16 \pm 0.25$ & $<0.01 *$ \\
\hline $\mathrm{FEV}_{1}(\%$ predicted $)$ & $45.92 \pm 15.05$ & $51.89 \pm 16.83$ & $5.97 \pm 9.15$ & $<0.01 *$ \\
\hline $\mathrm{iVaw}_{\text {FRC }}(\mathrm{mL})$ & $34.78 \pm 14.20$ & $36.28 \pm 12.87$ & $1.50 \pm 7.65$ & 0.19 \\
\hline $\mathrm{iVaw}_{\mathrm{TLC}}(\mathrm{mL})$ & $54.79 \pm 16.05$ & $56.49 \pm 16.32$ & $1.70 \pm 4.65$ & $0.02 *$ \\
\hline FRC (L) & $4.83 \pm 1.31$ & $4.62 \pm 1.17$ & $-0.22 \pm 0.58$ & $0.01 *$ \\
\hline iVlobe $_{\text {FRC }}(\mathrm{L})$ & $5.01 \pm 1.18$ & $4.75 \pm 1.10$ & $-0.25 \pm 0.6 \mathrm{I}$ & $<0.01 *$ \\
\hline TLC (L) & $6.72 \pm 1.42$ & $6.74 \pm 1.30$ & $0.02 \pm 0.60$ & 0.81 \\
\hline iVlobe $_{\text {TLC }}(\mathrm{L})$ & $6.47 \pm 1.18$ & $6.49 \pm 1.14$ & $0.02 \pm 0.4 \mathrm{I}$ & 0.76 \\
\hline Raw (kPa.s/L) & $0.7 I \pm 0.24$ & $0.63 \pm 0.34$ & $-0.10 \pm 0.29$ & $0.04^{*}$ \\
\hline $\mathrm{iRaw}_{\mathrm{FRC}}(\mathrm{kPa} \cdot \mathrm{s} / \mathrm{L})$ & $0.11 \pm 0.13$ & $0.06 \pm 0.08$ & $-0.04 \pm 0.12$ & $0.03^{*}$ \\
\hline $\mathrm{iRaw}_{\text {TLC }}(\mathrm{kPa} \cdot \mathrm{s} / \mathrm{L})$ & $0.04 \pm 0.03$ & $0.04 \pm 0.02$ & $-0.01 \pm 0.02$ & $0.03^{*}$ \\
\hline IC (L) & $1.96 \pm 0.59$ & $2.20 \pm 0.73$ & $0.14 \pm 0.27$ & $<0.01 *$ \\
\hline $\mathrm{RV}(\mathrm{L})$ & $3.87 \pm 1.23$ & $3.66 \pm 1.02$ & $-0.21 \pm 0.76$ & 0.07 \\
\hline $\mathrm{TCO}(\mathrm{mmol} / \mathrm{min} / \mathrm{kPa})$ & $3.52 \pm 1.50$ & $3.53 \pm 1.63$ & $0.06 \pm 0.67$ & 0.54 \\
\hline \multicolumn{5}{|c|}{ Patient-reported outcomes } \\
\hline SGRQ & $62.18 \pm \mid 5.31$ & $48.60 \pm 20.51$ & $-14.62 \pm 18.88$ & $<0.01 *$ \\
\hline $\mathrm{CCQ}$ & $3.40 \pm 1.20$ & $2.4 I \pm I .30$ & $-1.00 \pm 1.45$ & $<0.01 *$ \\
\hline CAT & $24.06 \pm 7.81$ & $18.23 \pm 8.13$ & $-5.83 \pm 11.28$ & $<0.01 *$ \\
\hline $\mathrm{mMRC}$ & $3.11 \pm 1.15$ & $2.43 \pm 1.17$ & $-0.67 \pm 1.23$ & $<0.01 *$ \\
\hline
\end{tabular}

Notes: Values are shown as mean $\pm \mathrm{SD}$. Correlations are reported as $p$-value and $r$. ${ }^{*} p$-value $<0.05$.

Abbreviations: CAT, COPD Assessment Test; CCQ, Clinical COPD Questionnaire; FEV , forced expiratory volume in I second; FRC, functional residual capacity; FRI, functional respiratory imaging; IC, inspiratory capacity; iRaw, airway resistance measured by FRI; iVaw, airway volume measured by FRI; iVlobe, lobar volume measured by FRI; mMRC, modified Medical Research Council dyspnea scale; Raw, airway resistance measured by plethysmography; RV, residual volume; SD, standard deviation; SGRQ, the Saint George's Respiratory Questionnaire; TCO, transfer factor for carbon monoxide; TLC, total lung capacity.

\section{FRI measures versus global patient changes}

We have taken a deeper look at 2 patients from those within this trial. Both were female GOLD stage III COPD patients, aged 57 and 62 years, with a smoking history of 35 and 20 packyears (hereafter they will be referred as patient 1 and patient 2 , respectively). Both patients present similar $\mathrm{FEV}_{1}$ values at baseline (39.0\% predicted and $40.5 \%$ predicted) as well as a similar change in $\mathrm{FEV}_{1}$ after recovering from an exacerbation (3.0\% predicted and $2.1 \%$ predicted). Even though the spirometry measurements are same for both patients, FRI parameters show clear differences between them. Table 4 and Figures 4 and 5 show the detailed overview of the FRI parameters.

At baseline, patient 1 is clearly hyperinflated, with a total lung volume of $140.6 \%$ predicted at TLC and $201.1 \%$ predicted at FRC in opposition to patient 2 ( $95.4 \%$ predicted at TLC and $118.5 \%$ predicted at FRC). When looking at changes in the FRI parameters, the increase in $\mathrm{FEV}_{1}$ is mainly associated with a decrease in iVlobe $(6.1 \%$ at TLC and $28.3 \%$ at FRC) for patient 1 and only partially associated with change in resistance. On the other hand, patient 2 has a slight increase in iVlobe (1.4\% at TLC and $4.9 \%$ at FRC), particularly in the upper lobes, but here the increase in $\mathrm{FEV}_{1}$ can be linked to larger iVaw values at FRC (+95.72\%) and a corresponding drop in resistance $(-90.80 \%)$.

These results suggest that FRI is a technique that can differentiate between patients with comparable spirometry measurements. Therefore, FRI may result in more precise diagnosis and treatment, particularly in heterogeneous diseases such as COPD.

\section{Discussion}

We showed that FRI is feasible in the acute setting of COPD exacerbations. It can demonstrate significant differences between exacerbation and stable state. The changes in FRI
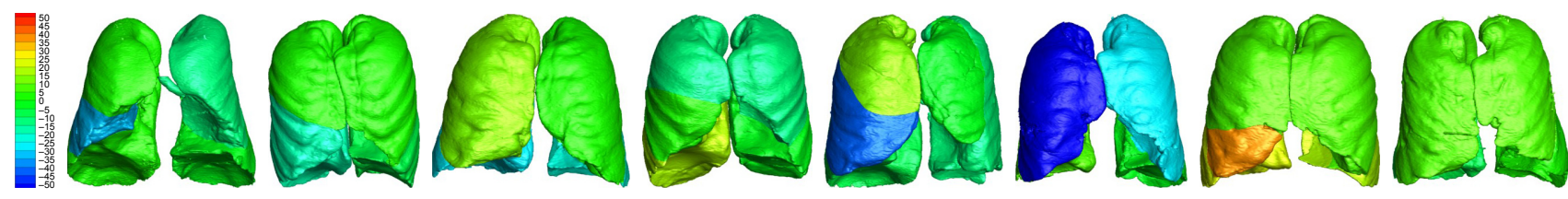

Figure 2 Variability in changes from exacerbation to stable state in iVlobe at TLC in individual patients (8 patients randomly selected). The scale represents the percent change in the volume of the different lung lobes at TLC; red represents an increase in volume, while blue represents a decrease.

Abbreviations: iVlobe, lobar volume measured by FRI; TLC, total lung capacity. 


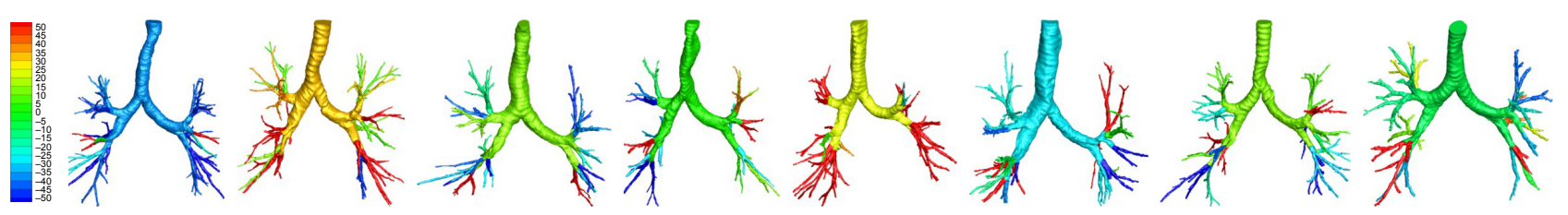

Figure 3 Variability in changes from exacerbation to stable state in siRaw at TLC in individual patients (8 patients randomly selected). The scale represents the percent change in the resistance of the different airway branches at TLC; red represents an increase in resistance, while blue represents a decrease.

Abbreviations: siRaw, specific airway resistance measured by FRI; TLC, total lung capacity.

correlate partially with changes in conventional lung function parameters and the quality of life. It clearly visualizes regional heterogeneity within patients and large differences between them.

Table 3 Correlation between change in FRI parameters and changes in classical pulmonary function tests and in the quality of life, from exacerbation to stable state

\begin{tabular}{|c|c|c|c|}
\hline $\begin{array}{l}\text { Change in pulmonary } \\
\text { function parameter or } \\
\text { patient-reported outcome }\end{array}$ & $\begin{array}{l}\text { Change in FRI } \\
\text { parameter (level } \\
\text { of measurement) }\end{array}$ & $r$ & $p$-value \\
\hline \multicolumn{4}{|l|}{ Primary end points } \\
\hline $\mathrm{FEV}_{1}(\mathrm{~L})$ & iVlobe (FRC) & 0.26 & 0.08 \\
\hline $\mathrm{FEV}_{1}(\mathrm{~L})$ & iVaw (TLC) & 0.21 & 0.16 \\
\hline FEV (L) & siVaw (FRC) & 0.34 & $0.02 *$ \\
\hline IC (L) & iVlobe (FRC) & 0.35 & 0.06 \\
\hline IC (L) & iVaw (TLC) & 0.09 & 0.62 \\
\hline IC (L) & siVaw (FRC) & 0.05 & 0.78 \\
\hline Raw & iRaw (FRC) & 0.33 & $0.04 *$ \\
\hline sRaw & siRaw (TLC) & 0.13 & 0.42 \\
\hline \multicolumn{4}{|l|}{ Secondary end points } \\
\hline SGRQ & $\mathrm{FEV}_{1}(\mathrm{~L})$ & 0.24 & 0.11 \\
\hline SGRQ & iVlobe (FRC) & 0.14 & 0.38 \\
\hline SGRQ & iVaw (TLC) & 0.12 & 0.45 \\
\hline SGRQ & siVaw (FRC) & 0.17 & 0.26 \\
\hline SGRQ & siRaw (TLC) & 0.28 & 0.07 \\
\hline $\mathrm{CCQ}$ & $\mathrm{FEV}_{1}(\mathrm{~L})$ & 0.20 & 0.17 \\
\hline $\mathrm{CCQ}$ & iVlobe (FRC) & 0.24 & 0.11 \\
\hline $\mathrm{CCQ}$ & iVaw (TLC) & 0.12 & 0.41 \\
\hline $\mathrm{CCQ}$ & siVaw (FRC) & 0.14 & 0.34 \\
\hline $\mathrm{CCQ}$ & siRaw (TLC) & 0.36 & $0.01 *$ \\
\hline CAT & FEV (L) & 0.38 & $0.01 *$ \\
\hline CAT & iVlobe (FRC) & 0.26 & 0.08 \\
\hline CAT & iVaw (TLC) & 0.26 & 0.08 \\
\hline CAT & siVaw (FRC) & 0.17 & 0.24 \\
\hline CAT & siRaw (TLC) & 0.42 & $<0.01 *$ \\
\hline $\mathrm{mMRC}$ & $\mathrm{FEV}_{1}(\mathrm{~L})$ & 0.31 & $0.04 *$ \\
\hline mMRC & iVlobe (FRC) & 0.12 & 0.45 \\
\hline $\mathrm{mMRC}$ & iVaw (TLC) & 0.17 & 0.25 \\
\hline $\mathrm{mMRC}$ & siVaw (FRC) & 0.36 & $0.01 *$ \\
\hline $\mathrm{mMRC}$ & siRaw (TLC) & 0.30 & $0.045^{*}$ \\
\hline
\end{tabular}

Notes: Correlations are reported as $p$-value and $r .{ }^{*} p$-value $<0.05$.

Abbreviations: CAT, COPD Assessment Test; CCQ, Clinical COPD Questionnaire; $\mathrm{FEV}_{\text {, }}$, forced expiratory volume in I second; $\mathrm{FRC}$, functional residual capacity; FRI, functional respiratory imaging; IC, inspiratory capacity; iRaw, airway resistance measured by FRI; iVaw, airway volume measured by FRI; iVlobe, lobar volume measured by FRI; mMRC, modified Medical Research Council dyspnea scale; Raw, airway resistance measured by plethysmography; RV, residual volume; SGRQ, the Saint George's Respiratory Questionnaire; sRaw, specific airway resistance measured by pulmonary function test; siRaw, specific airway resistance measured by FRI; siVaw, specific airway volumes measured by FRI; TCO, transfer factor for carbon monoxide; TLC, total lung capacity.
FRI allows individualized measurements of patients with an acute exacerbation of COPD and among others visualizes the marked differences between patients. It does not measure a pulmonary function test with $\mathrm{CT}$, but it measures different parameters. The parameters, hyperinflation, airway diameter, and air resistance, specifically of the lungs and segments within the lungs, are measured without bias from the oropharynx, eg, cough and oxygen masks. Importantly, the FRI parameters improve after the recovery of exacerbation. Since a CT scan can be performed during an acute event, even when conventional pulmonary function testing is not well possible, these findings might influence treatment decisions in the future, both for the group of acute exacerbations and for individuals.

Next to the feasibility of FRI during exacerbations, this study also showed the ability to measure different regions of the lung during COPD exacerbations. One could have thought that volume and resistance changes during exacerbations were evenly distributed throughout the lung; however, our study showed in a small group marked changes between different regions. Next, changes in $\mathrm{FEV}_{1}$ after an exacerbation are not always determined by changes in the larger airways, but can also be driven by changes in the smaller airways, and

Table 4 Changes in functional respiratory imaging parameters from exacerbation to stable state in 2 individual patients with similar changes in classical pulmonary function tests

\begin{tabular}{|c|c|c|c|c|}
\hline \multirow[t]{2}{*}{ Parameters } & \multicolumn{2}{|l|}{ Patient I } & \multicolumn{2}{|l|}{ Patient 2} \\
\hline & Baseline & $\begin{array}{l}\text { Change } \\
\text { (\%) }\end{array}$ & Baseline & $\begin{array}{l}\text { Change } \\
\text { (\%) }\end{array}$ \\
\hline $\mathrm{FEV}_{\text {, }}$ & $0.82 \mathrm{~L}$ & +7.32 & $0.77 \mathrm{~L}$ & +9.09 \\
\hline $\mathrm{iVaw}_{\mathrm{FRC}}$ & $32.41 \mathrm{~mL}$ & -6.29 & $7.37 \mathrm{~mL}$ & +95.72 \\
\hline $\mathrm{iVaw}_{\text {TLC }}$ & $42.20 \mathrm{~mL}$ & +14.79 & $29.13 \mathrm{~mL}$ & +3.06 \\
\hline FRC & $4.86 \mathrm{~L}$ & -9.88 & $2.92 \mathrm{~L}$ & +12.67 \\
\hline iVlobe $_{F R C}$ & $201.13 \%$ predicted & -28.33 & I I $8.52 \%$ predicted & +4.87 \\
\hline TLC & $6.22 \mathrm{~L}$ & -0.96 & $4.59 \mathrm{~L}$ & +3.92 \\
\hline iVlobe $_{\text {TLC }}$ & I 40.62\% predicted & -6.14 & $95.35 \%$ predicted & +1.43 \\
\hline Raw & $0.44 \mathrm{I} \mathrm{kPa} \cdot \mathrm{s} / \mathrm{L}$ & +20.18 & $1.060 \mathrm{kPa} \cdot \mathrm{s} / \mathrm{L}$ & -18.96 \\
\hline $\mathrm{iRaw}_{\mathrm{FRC}}$ & $0.05 \mathrm{kPa} \cdot \mathrm{s} / \mathrm{L}$ & +5.51 & $0.17 \mathrm{kPa} \cdot \mathrm{s} / \mathrm{L}$ & -89.90 \\
\hline $\mathrm{iRaw}_{\text {TLC }}$ & $0.09 \mathrm{kPa} \cdot \mathrm{s} / \mathrm{L}$ & -51.35 & $0.05 \mathrm{kPa} \cdot \mathrm{s} / \mathrm{L}$ & -0.82 \\
\hline
\end{tabular}

Abbreviations: $\mathrm{FEV}$, forced expiratory volume in I second; FRC, functional residual capacity; iRaw, airway resistance measured by FRI; iVaw, airway volume measured by FRI; iVlobe, lobar volume measured by FRI; Raw, airway resistance measured by plethysmography; TLC, total lung capacity. 


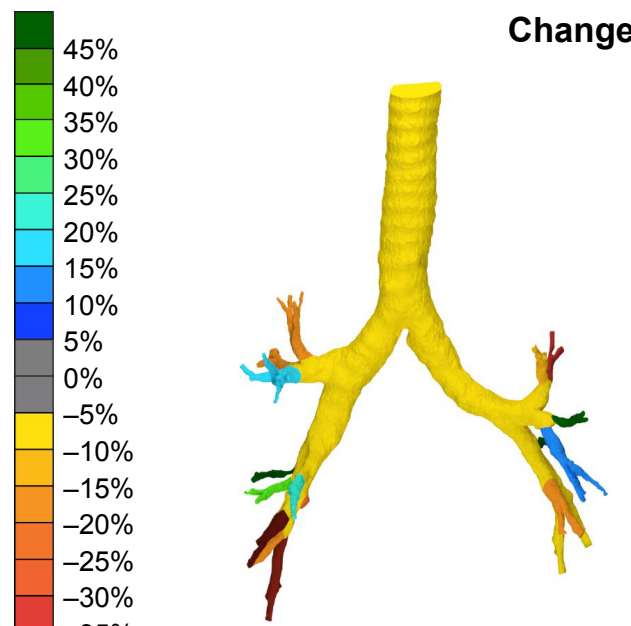

Patient 1
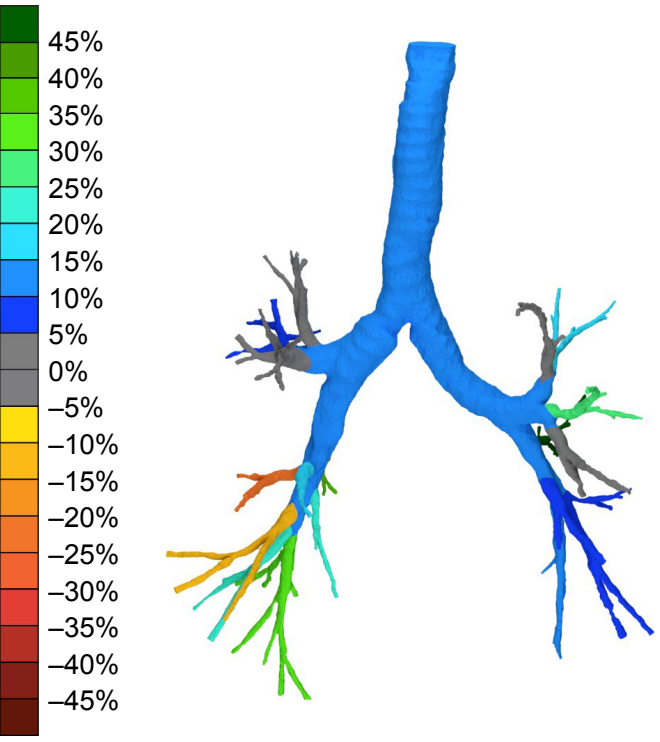

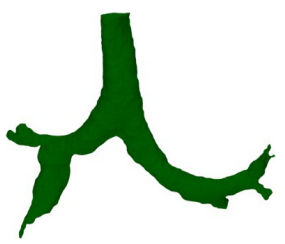

FRC

Figure $4 \mathrm{FRI}$ images of 2 patients displaying the changes in airway volume. The scale represents the percent change in the volume of the different airway branches at the different lung levels. Green represents an improvement, while red represents a worsening. The airway volume is the volume of the lumen of the airways and does thus represent the volume of air inside the airways. The airways are measured starting from the trachea at the top of the sternum up to the point where no distinction can be made between the intraluminal and alveolar air. This is where the airway diameter is around I-2 mm. One can infer that an increase in airway volume means bronchodilation has occurred. ${ }^{18}$

Abbreviations: FRC, functional residual capacity; FRI, functional respiratory imaging; TLC, total lung capacity.

this influence is different in different subjects. To gain a more complete overview about this heterogeneity and its clinical implications, a study of larger sample size with a treatment algorithm is required. However, one could imagine that this new described intrapatient heterogeneity of the lung function can be of interest for future practitioners, since treating different lung regions might require different strategies. ${ }^{12}$ Could regional guided treatment be of use during exacerbations as well? Are those exacerbations with increased airway resistance the ones requiring a more frequent, higher dosed nebulizer treatment, such as asthma?
Thus far, no specific therapies for local differences in resistance have made it into daily clinical practice. During exacerbations, the presence and magnitude of effects of bronchodilators on resistance are not routinely assessed at all. FRI allows for measurement of airway resistance at several levels and has the potential to demonstrate and visualize differences between severity and location of individual patients. It was not possible to detect whether changes are caused by mucus, airway wall thickening, muscle contraction, or other causes. Differences between the individuals can be expected to be high, and cluster analysis of larger patient groups will 


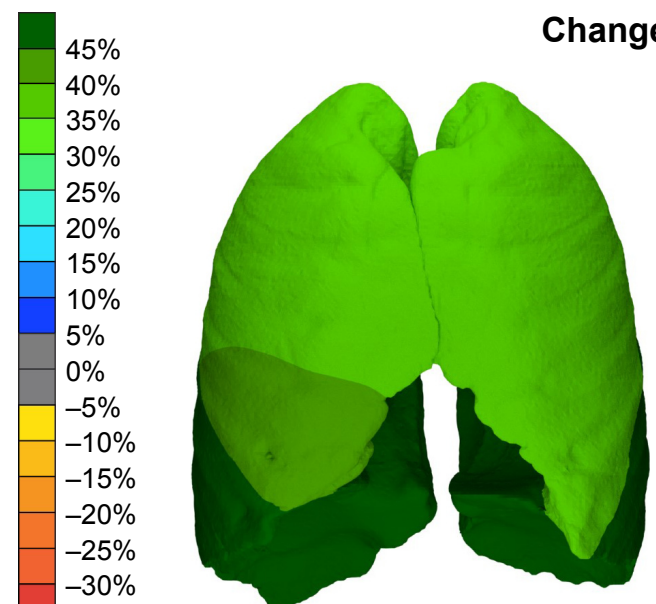

Patient 1
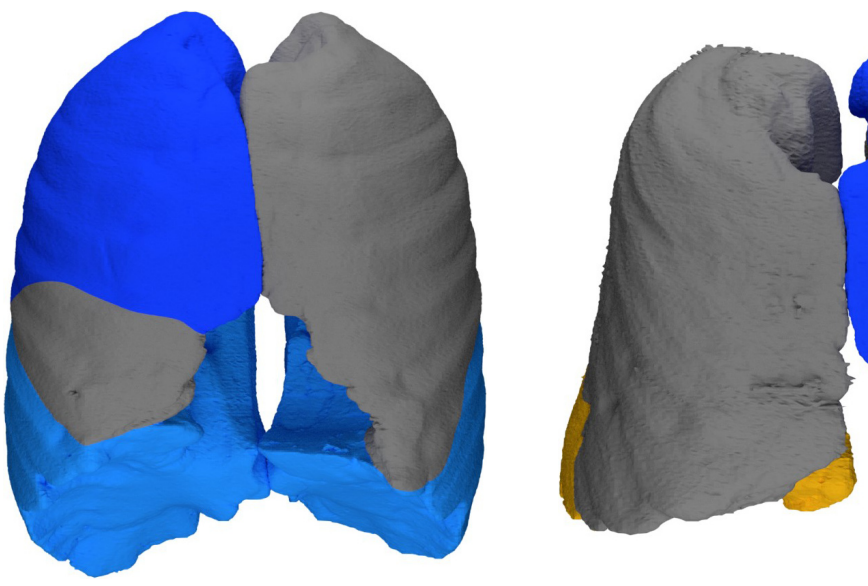

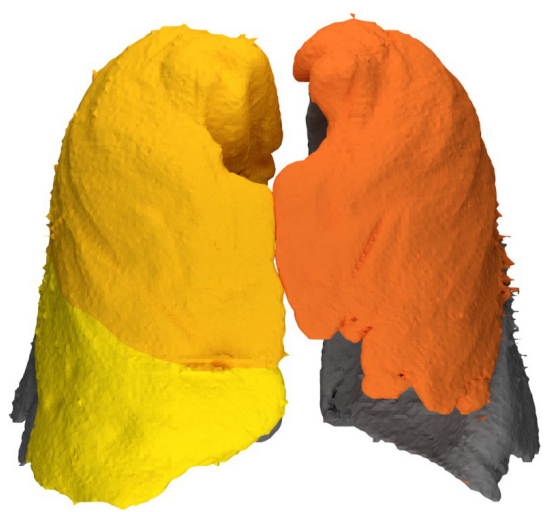

Patient 2
FRC

TLC

Figure 5 FRI images of 2 patients displaying the changes in lobar volume. The scale represents the percent change in the volume of the different airway branches at the different lung levels; green represents an improvement, while red represents a worsening.

Abbreviations: FRC, functional residual capacity; FRI, functional respiratory imaging; TLC, total lung capacity.

help to identify the best strategy based on airway resistance during exacerbations.

This study shows the feasibility of measuring FRI parameters during exacerbations and compared the results with stable state. In clinical routine, the feasibility of lung function testing in patients admitted with an exacerbation of COPD is limited due to several reasons such as staff attendance, the absence of plethysmography, and the clinical condition of the patient. Since a CT scan can be performed during an acute event, even in patients in severe respiratory distress when conventional pulmonary function testing is not well possible, FRI might have a specific future role in the clinical assessment of COPD exacerbation. Costs and radiation however need to be considered.
FRI could be used to assess the parameters of increased hyperinflation during the exacerbation. Then, FRI could aid in individualizing treatment, above all of the bronchodilators. In patients who, after initial bronchodilation, show persistent hyperinflation, the effect of additional bronchodilation should be examined. Based on the distribution of the hyperinflation, bronchodilator device selection may be performed: for instance, regular nebulizers in case of central hyperinflation and pressurized metered-dose inhalers with spacer in more peripheral hyperinflation. Another potential strategy could be the selection of the type of bronchodilator. In stable COPD, long-acting bronchodilators have replaced short-acting bronchodilators in patients with a more severe hyperinflation. We speculate that, during exacerbations, FRI can be used 
to treat patients with more hyperinflation with long-acting bronchodilators compared with the current standard treatment of short-acting bronchodilators in less hyperinflated patients. ${ }^{6}$ In addition, different physiotherapeutic strategies, eg, to regulate breathing next to sputum evacuation, can be considered for this group, and in the long run perhaps noninvasive lung volume reduction techniques, currently tested only in stable, though severely obstructed patients, could perhaps be attempted, as guided by FRI. ${ }^{27,28}$

It would be of great clinical importance if FRI measurements can also aid in delineating the absence of hyperinflation and small airway dysfunction, a markedly different phenotype of exacerbation. Some of these patients suffer predominantly from fear and should be reassured and treated for their anxiety, as opposed to more bronchodilators. The latter by inducing tachycardia and giving false hopes might even augment fear. We speculate that FRI especially in combination with other biomarkers such as eosinophilia or viral and bacterial diagnosis will allow more precise assessments in acute exacerbations, potentially unlocking precision treatment.

This study has several strengths and weaknesses that should be mentioned. It has been performed in different centers in different countries each with their own local radiologic and pulmonary function equipment making the applicability of its results broader: it is feasible in several different clinical settings. The correlations between the standard pulmonary function measurements and the FRI measurements were relatively low, which should not be a surprise given the difficulty of assessing them during exacerbations. During exacerbations, all measurements, including classical lung function, will be more variable than during stable state, lowering all correlations. At current, the variability in all these tests, including spirometry, during exacerbations is largely unknown and should be examined. The usefulness of all tests to adequately guide clinical decisions should be assessed, as much of the FRI measurements as of, for instance, $\mathrm{FEV}_{1}$, which is not routinely performed during acute exacerbations probably for good reason. Survival, hospital readmission, length of stay, and response to therapy should be considered as end points in future trials assessing treatment strategies.

\section{Conclusion}

In summary, we have shown for the first time that FRI based on HRCT measurements is feasible also in patients with an acute exacerbation of COPD. During the resolution of the exacerbation, the parameters indeed improve considerably. The changes do correlate to some degree with changes in conventionally measured parameters such as change in $\mathrm{FEV}_{1}$, resistance, and hyperinflation, as well as with PROs, but also provide additional information. The technique clearly visualizes the marked variability within and between individuals in ventilation and resistance during exacerbations. Information about regional differences in patients and heterogeneity between patients can now be obtained.

\section{Acknowledgment}

The study was funded by FLUIDDA nv, Kontich, Belgium, and GlaxoSmithKline R\&D (GSK), Stevenage, UK, with an unrestricted educational grant.

\section{Disclosure}

WHVG received an ERS Fellowship STRTF 2016 and reports a grant from Novartis to the institution for an investigator-initiated trial outside of the submitted work. WV reports grants from GSK, during the conduct of the study, and personal fees from FLUIDDA nv, outside of the submitted work. JDB is the founder and shareholder of FLUIDDA nv, a company that develops and markets part of the technology described in this paper. AC reports a grant from GSK, outside of the submitted work. OSU reports grants from AstraZeneca, GSK, Prosonix, and Edmond Pharma; personal fees from Boehringer Ingelheim, Aerocrine, Napp, Mundipharma, Sandoz, Takeda, Zentiva, and Cipla; and grants and personal fees from Chiesi, outside of the submitted work. CVH reports a grant from FLUIDDA nv, during the conduct of the study and also outside of the submitted work. HAMK reports that his institution has received a per patient fee for participation in the trial reported from FLUIDDA nv. BH, MP, and WDB report no conflicts of interest in this work.

\section{References}

1. Global Initiative for Chronic Obstructive Lung Disease. Global Strategy for the Diagnosis, Management and Prevention of COPD; 2016. Available from: http:/goldcopd.org/global-strategy-diagnosis-managementprevention-copd-2016/. Accessed September 25, 2017.

2. Leidy NK, Wilcox TK, Jones PW, et al. Standardizing measurement of chronic obstructive pulmonary disease exacerbations. Reliability and validity of a patient-reported diary. Am J Respir Crit Care Med. 2011;183(3): 323-329

3. Kocks JW, van den Berg JW, Kerstjens HA, et al. Day-to-day measurement of patient-reported outcomes in exacerbations of chronic obstructive pulmonary disease. Int J Chron Obstruct Pulmon Dis. 2013;8: 273-286.

4. Aaron SD. Management and prevention of exacerbations of COPD. BMJ. 2014;349:g5237.

5. Parker CM, Voduc N, Aaron SD, Webb KA, O’Donnell DE. Physiological changes during symptom recovery from moderate exacerbations of COPD. Eur Respir J. 2005;26(3):420-428.

6. van Geffen WH, Douma WR, Slebos DJ, Kerstjens HA. Bronchodilators delivered by nebuliser versus pMDI with spacer or DPI for exacerbations of COPD. Cochrane Database Syst Rev. 2016;(8):CD011826.

7. Lopez-Campos JL, Agusti A. Heterogeneity of chronic obstructive pulmonary disease exacerbations: a two-axes classification proposal. Lancet Respir Med. 2015;3(9):729-734. 
8. van Geffen WH, Bruins M, Kerstjens HA. Diagnosing viral and bacterial respiratory infections in acute COPD exacerbations by an electronic nose: a pilot study. J Breath Res. 2016;10(3):036001.

9. van Geffen WH, Slebos DJ, Kerstjens HA. Hyperinflation in COPD exacerbations. Lancet Respir Med. 2015;3(12):e43-e44.

10. Mahler DA, O’Donnell DE. Recent advances in dyspnea. Chest. 2015; 147(1):232-241.

11. O'Donnell DE, Laveneziana P. The clinical importance of dynamic lung hyperinflation in COPD. COPD. 2006;3(4):219-232.

12. Shah PL, Herth FJ, van Geffen WH, Deslee G, Slebos DJ. Lung volume reduction for emphysema. Lancet Respir Med. 2017;5(2):147-156.

13. Cooper CB. The connection between chronic obstructive pulmonary disease symptoms and hyperinflation and its impact on exercise and function. Am J Med. 2006;119(10 Suppl 1):21-31.

14. Rossi A, Aisanov Z, Avdeev S, et al. Mechanisms, assessment and therapeutic implications of lung hyperinflation in COPD. Respir Med. 2015;109(7):785-802.

15. Stevenson NJ, Walker PP, Costello RW, Calverley PM. Lung mechanics and dyspnea during exacerbations of chronic obstructive pulmonary disease. Am J Respir Crit Care Med. 2005;172(12):1510-1516.

16. Taube C, Lehnigk B, Paasch K, Kirsten DK, Jorres RA, Magnussen H. Factor analysis of changes in dyspnea and lung function parameters after bronchodilation in chronic obstructive pulmonary disease. Am J Respir Crit Care Med. 2000;162(1):216-220.

17. Santus P, Radovanovic D, Henchi S, et al. Assessment of acute bronchodilator effects from specific airway resistance changes in stable COPD patients. Respir Physiol Neurobiol. 2014;197:36-45.

18. De Backer JW, Vos WG, Gorle CD, et al. Flow analyses in the lower airways: patient-specific model and boundary conditions. Med Eng Phys. 2008;30(7):872-879.

19. De Backer JW, Vos WG, Vinchurkar SC, et al. Validation of computational fluid dynamics in CT-based airway models with SPECT/CT. Radiology. 2010;257(3):854-862.
20. De Backer LA, Vos W, De Backer J, Van Holsbeke C, Vinchurkar S, De Backer W. The acute effect of budesonide/formoterol in COPD: a multi-slice computed tomography and lung function study. Eur Respir J. 2012;40(2):298-305.

21. Vos W, De Backer J, Poli G, et al. Novel functional imaging of changes in small airways of patients treated with extrafine beclomethasone/ formoterol. Respiration. 2013;86(5):393-401.

22. Hajian B, De Backer J, Vos W, Van Holsbeke C, Clukers J, De Backer W. Functional respiratory imaging (FRI) for optimizing therapy development and patient care. Expert Rev Respir Med. 2016;10(2): 193-206.

23. Vos W, Hajian B, De Backer J, et al. Functional respiratory imaging to assess the interaction between systemic roflumilast and inhaled ICS/LABA/LAMA. Int J Chron Obstruct Pulmon Dis. 2016;11: 263-271.

24. Global Initiative for Chronic Obstructive Lung Disease. Global Strategy for the Diagnosis, Management and Prevention of COPD. Global Initiative for Chronic Obstructive Lung Disease 2010; 2010. Available from: www.goldcopd.org

25. Jones PW, Beeh KM, Chapman KR, Decramer M, Mahler DA, Wedzicha JA. Minimal clinically important differences in pharmacological trials. Am J Respir Crit Care Med. 2014;189(3):250-255.

26. Miller MR, Hankinson J, Brusasco V, et al. Standardisation of spirometry. Eur Respir J. 2005;26(2):319-338.

27. van Geffen WH, Herth FJ, Deslee G, Slebos DJ, Shah PL. Lung volume reduction for emphysema - authors' reply. Lancet Respir Med. 2017;5(7):e24.

28. van Geffen WH, Kerstjens HAM, Slebos DJ. Emerging bronchoscopic treatments for chronic obstructive pulmonary disease. Pharmacol Ther. 2017;179:96-101.
International Journal of COPD

\section{Publish your work in this journal}

The International Journal of COPD is an international, peer-reviewed journal of therapeutics and pharmacology focusing on concise rapid reporting of clinical studies and reviews in COPD. Special focus is given to the pathophysiological processes underlying the disease, intervention programs, patient focused education, and self management protocols.

\section{Dovepress}

This journal is indexed on PubMed Central, MedLine and CAS. The manuscript management system is completely online and includes a very quick and fair peer-review system, which is all easy to use. Visit http://www.dovepress.com/testimonials.php to read real quotes from published authors. 